\title{
On the action of pseudo-differential operators on Gevrey spaces
}

\author{
Baptiste Morisse *
}

October 1, 2018

\begin{abstract}
In this paper we study the action of pseudo-differential operators acting on Gevrey spaces. We introduce classes of classical symbols with spatial Gevrey regularity. As the spatial Gevrey regularity of a symbol $p(\cdot, \xi)$ may depend on the frequency $\xi$, the action of the associated pseudo-differential operator op $(p)$ may induce a loss of regularity. The proof is based on a para-product decomposition.
\end{abstract}

\section{Contents}

1 Introduction

2 Classes of Gevrey regular symbols 2

2.1 Gevrey spaces . . . . . . . . . . . . . . . . . 2 2

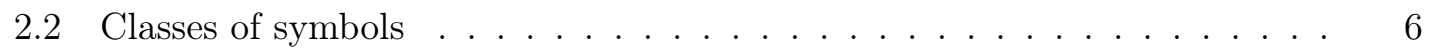

3 Conjugation of a Gevrey function 6

4 Action of pseudo-differential operators on Gevrey spaces 8

5 A conjugation Lemma for operators

\section{Introduction}

This paper is devoted to the study of a class of pseudo-differential operators acting in Gevrey spaces. We assume that the operators have symbols which are Gevrey regular in the spatial variable $x \in \mathbb{R}^{d}$ and satisfy estimates in $(x, \xi)$ derivatives which are analogous to the ones enjoyed by symbols of the classical classes $S_{\rho, \delta}^{m}$. These symbols are precisely defined in Section 2. We give three results:

${ }^{*}$ School of Mathematics, Cardiff University - morisseb@cardiff.ac.uk. The author is supported by the EPSRC grant "Quantitative Estimates in Spectral Theory and Their Complexity" (EP/N020154/1). The author thanks his PhD advisor Benjamin Texier for all the remarks on this work, and Nicolas Lerner for interesting discussions. 
- The first, Proposition 3.1, states that if a function $F$ belongs to $H^{m} \mathcal{G}_{\tau}^{\sigma}$ (a Gevrey space with Sobolev correction, defined in Section 2), then the operator $e^{\tau D^{\sigma}} F e^{-\tau D^{\sigma}}$ acts continuously in $H^{m}\left(\mathbb{R}^{d}\right)$. This result appeared in slightly different form in the article [BMM13] by Bedrossian, Masmoudi and Mouhot (see Lemma 3.3 therein). Its proof relies on a para-product decomposition and precise triangle-like inequalities in the spirit of [BMM13].

- Our second and main result, Theorem 4.2, describes the action of operators with symbols in $\mathrm{S}_{\rho, \delta}^{0} \mathrm{G}_{R}^{s}$ (classical symbols with Gevrey regularity, defined in Section 2 ) on Gevrey spaces. The proof relies again on a para-product decomposition.

- The third result is Lemma 5.1. Here we give precise bounds for the symbol of $e^{\tau D^{\sigma}} \operatorname{op}(p) e^{-\tau D^{\sigma}}$, where $p$ belongs to $\mathrm{S}_{\rho, 0}^{0} \mathrm{G}_{R}^{s}$. This completes Lemma 7.1 of the article [CNR] by Colombini, Nishitani and Rauch.

A classical reference on Gevrey spaces is Rodino's book Rod93. See also the paper HR01 by Hua and Rodino, where slightly less general classes of symbols are studied. Questions about the action of pseudo-differential operators in Gevrey spaces naturally arise from the study of the Gevrey well-posedness of the Cauchy problem for first-order systems. The aforementioned article [CNR] focuses on Gevrey well-posedness, and so does our own line of research [Mor16a], Mor16b], Mor17a] and [Mor17b].

\section{Classes of Gevrey regular symbols}

\subsection{Gevrey spaces}

We start by two definitions of Gevrey spaces, one on the spatial side, the other on the Fourier side.

Definition 2.1 (Gevrey spaces: the spatial viewpoint). For any $s \in[1, \infty)$, we define $G_{R}^{s}$ to be the space of smooth functions $f$ such that, for any compact set $K$ of $\mathbb{R}^{d}$, there are two positive constants $C_{K}$ and $R_{K}$ for which there holds

$$
\left|\partial_{x}^{\alpha} f\right|_{L^{\infty}(K)} \leq C_{K} R_{K}^{|\alpha|}|\alpha| !^{s} \quad, \quad \forall \alpha \in \mathbb{N}^{d} .
$$

We call s the Gevrey (regularity) index, and $R_{K}^{-1}$ the Gevrey radius.

For $B$ a compact set of $\mathbb{R}^{d}$ and $R>0$ being fixed, we define $G_{R}^{s}(B)$ the space of smooth functions $f$ compactly supported on $B$ and being in $G^{s}$. That is, there is a positive constant $C>0$ for which there holds

$$
\left|\partial_{x}^{\alpha} f\right|_{L^{\infty}(B)} \leq C R^{|\alpha|}|\alpha|^{! s} \quad, \quad \forall \alpha \in \mathbb{N}^{d} .
$$

The space $G_{R}^{s}(B)$ can be associated with the norm defined by

$$
|f|_{s, R}=\sup _{\alpha \in \mathbb{N}^{d}}\left|\partial_{x}^{\alpha} f\right|_{L^{\infty}(B)}\left(R^{|\alpha|}|\alpha| !^{s}\right)^{-1}
$$

We define

$$
\langle\xi\rangle=\left(1+|\xi|^{2}\right)^{1 / 2} \quad, \quad \forall \xi \in \mathbb{R}^{d} .
$$


Definition 2.2 (Gevrey spaces: the Fourier viewpoint). For any $\sigma \in(0,1]$ and $\tau>0$, we define $\mathcal{G}_{\tau}^{\sigma}$ to be the space of functions $f \in L^{2}$ such that $\exp \left(\tau\langle\cdot\rangle^{\sigma}\right) \hat{f}$ is in $L^{2}$. The associated norm is defined by

$$
|f|_{\sigma, \tau}=\left|e^{\tau\langle\cdot\rangle^{\sigma}} \widehat{f}\right|_{L^{2}} .
$$

We call $\tau$ the Gevrey radius and $\sigma$ the Gevrey (regularity) index.

Both previous definitions of Gevrey functions are linked, as shown by the following classical result (see [Rod93]):

Proposition $2.1\left(G_{R}^{s}(B)\right.$ is included in $\left.\mathcal{G}_{\tau}^{1 / s}\right)$. For any compact set $B$ of $\mathbb{R}^{d}$, the space $G_{R}^{s}(B)$ is included in the space $\mathcal{G}_{\tau}^{\sigma}$ for $\sigma=1 / s$ and $\tau<s R^{-1 / s}$. Moreover there holds

$$
|u|_{\sigma, \tau} \leq|B|^{1 / 2} C\left(\tau s^{-1} R^{1 / s}\right)|u|_{s, R}
$$

with

$$
C(y) \lesssim \frac{1}{1-y} P\left(\frac{y}{1-y}\right)
$$

where $P$ is a polynomial with degree at most $\lceil(3 s-1) / 2\rceil$, and the implicit constant depends only on the Gevrey index s.

Proof. First we write $|u|_{\sigma, \tau}=e^{\tau}\left|e^{\tau\left(\langle\xi\rangle^{\sigma}-1\right)} \widehat{u}(\xi)\right|_{L^{2}}$ and there holds

$$
|u|_{\sigma, \tau} \leq e^{\tau} \sum_{n \geq 0} \frac{\tau^{n}}{n !}\left|\left(\langle\xi\rangle^{\sigma}-1\right)^{n} \widehat{u}(\xi)\right|_{L^{2}} .
$$

Next we compute $\langle\xi\rangle^{\sigma}-1=\sigma \int_{0}^{1}\langle t \xi\rangle^{\sigma-2} t|\xi|^{2} d t$ which implies, as $\sigma-2<0$, that

$$
\begin{aligned}
\langle\xi\rangle^{\sigma}-1 & \leq \sigma \int_{0}^{1}(t|\xi|)^{\sigma-2} t|\xi|^{2} d t \\
& \leq|\xi|^{\sigma} \int_{0}^{1} \sigma t^{\sigma-1} d t \\
& \leq|\xi|^{\sigma} .
\end{aligned}
$$

Thus there holds

$$
|u|_{\sigma, \tau} \leq e^{\tau} \sum_{n \geq 0} \frac{\tau^{n}}{n !} \|\left.\left.\xi\right|^{n \sigma} \widehat{u}(\xi)\right|_{L^{2}} .
$$

Let $n$ be given in the following. By the support of $u$ and inequalities (2.1), there holds

$$
\left.\left.|| \xi\right|^{n \sigma} \widehat{u}(\xi)\right|_{L^{2}} \leq|B|^{1 / 2}|u|_{s, R} R^{m} m !^{s}
$$

for any $m \geq n \sigma$. Thus

$$
\frac{\tau^{n}}{n !} \|\left.\left.\xi\right|^{n \sigma} \widehat{u}(\xi)\right|_{L^{2}} \leq \frac{m !^{s}}{n !}|B|^{1 / 2}|u|_{s, R} \tau^{n} R^{m} .
$$


Using Stirling's formula with $m<n \sigma+1$, there is $\delta>0$ such that

$$
\begin{aligned}
\frac{m !^{s}}{n !} & \leq(1+\delta)^{s} \frac{1}{n !}\left(\frac{n \sigma+1}{e}\right)^{(n \sigma+1) s}(2 \pi(n \sigma+1))^{s / 2} \\
& \leq(1+\delta)^{2 s}\left(\frac{n \sigma+1}{e}\right)^{(n \sigma+1) s}\left(\frac{n}{e}\right)^{-n}(2 \pi(n \sigma+1))^{s / 2}(2 \pi n)^{-1 / 2}
\end{aligned}
$$

As $s \sigma=1$, there holds

$$
\left(\frac{n \sigma+1}{e}\right)^{(n \sigma+1) s}\left(\frac{n}{e}\right)^{-n} \leq \sigma^{n}(n \sigma+1)^{s} e^{1 / \sigma-s} .
$$

This implies finally

$$
\frac{m !^{s}}{n !} \lesssim \sigma^{n} n^{(3 s-1) / 2}
$$

hence

$$
\left.\left.\frac{\tau^{n}}{n !}|| \xi\right|^{n \sigma} \widehat{u}(\xi)\right|_{L^{2}} \lesssim|B|^{1 / 2}|u|_{s, R} n^{(3 s-1) / 2}\left(\sigma \tau R^{\sigma}\right)^{n} .
$$

It now suffices to sum in $n \in \mathbb{N}$.

We recall here some useful inequalities when dealing with Gevrey spaces $\mathcal{G}_{\tau}^{\sigma}$.

\section{Lemma 2.2.}

1. Let $\sigma \in(0,1)$, $\xi$ and $\eta$ in $\mathbb{R}^{d}$ such that $|\xi-\eta| \leq \frac{1}{K}|\eta|$ for some $K>1$. Then

$$
\left|\langle\xi\rangle^{\sigma}-\langle\eta\rangle^{\sigma}\right| \leq\left(K^{\sigma}-(K-1)^{\sigma}\right)\langle\xi-\eta\rangle^{\sigma}
$$

Note that $K^{\sigma}-(K-1)^{\sigma}<1$ for any $K>1$.

2. Let $\sigma \in(0,1)$, $\xi$ and $\eta$ in $\mathbb{R}^{d}$ such that $\frac{1}{K}|\xi-\eta| \leq|\eta| \leq K|\xi-\eta|$ for some $K>1$. Then

$$
\langle\xi\rangle^{\sigma} \leq\langle\eta\rangle^{\sigma}+c^{\prime}\langle\xi-\eta\rangle^{\sigma}
$$

for some $c^{\prime} \in(0,1)$ depending on $K$.

3. For any $\xi \in \mathbb{R}^{d}, \sigma \in(0,1), \tau>0$ and $m \geq 0$, there holds

$$
\langle\xi\rangle^{m} \lesssim \tau^{-m / \sigma} e^{\tau\langle\xi\rangle^{\sigma}}
$$

Remark 1. Note that the first point in the previous Lemma does not hold when $\sigma=1$, i.e. in the analytic regularity.

Proof. Denote $f(t)=\langle\eta+t(\xi-\eta)\rangle^{\sigma}$. The function $f$ is differentiable on $[0,1]$, and there holds

$$
f(1)-f(0)=\sigma(\xi-\eta) \cdot \int_{0}^{1}(\eta+t(\xi-\eta))\langle\eta+t(\xi-\eta)\rangle^{\sigma-2} d t .
$$


As $|\xi-\eta| \leq \frac{1}{K}|\eta|$, there holds $|\eta+t(\xi-\eta)| \geq(K-t)|\xi-\eta|$ hence

$$
\begin{aligned}
|f(1)-f(0)| & \leq \sigma|\xi-\eta| \int_{0}^{1}\langle\eta+t(\xi-\eta)\rangle^{\sigma-1} d t \\
& \leq \sigma\langle\xi-\eta\rangle^{\sigma} \int_{0}^{1}(K-t)^{\sigma-1} d t
\end{aligned}
$$

which is (2.7). We now turn to the proof of $(2.8)$, from $|\eta| \geq K^{-1}|\xi-\eta|$ we deduce

$$
1+|\eta|^{2} \geq 1+K^{-2}|\xi-\eta|^{2}=K^{-2}\left(K^{2}+|\xi-\eta|^{2}\right) \geq K^{-2}\langle\xi-\eta\rangle^{2}
$$

where we used $K>1$. Thus, since $0<\sigma$,

$$
\langle\eta\rangle^{\sigma} \geq K^{-\sigma}\langle\xi-\eta\rangle^{\sigma}
$$

This implies

$$
\langle\eta\rangle^{\sigma}+\langle\xi-\eta\rangle^{\sigma} \geq\left(1+K^{-\sigma}\right)\langle\xi-\eta\rangle^{\sigma}
$$

Now assume in addition

$$
|\xi| \leq c|\xi-\eta|, \quad \text { for some } c>0 \text {. }
$$

If 2.10 holds with some $c \geq 1$, then it holds a fortiori with $c>1$. Thus we may assume 2.10 for some $c>1$, and then

$$
\langle\xi\rangle \leq c\langle\xi-\eta\rangle,
$$

so that

$$
\langle\xi\rangle^{\sigma} \leq c^{\sigma}\langle\xi-\eta\rangle^{\sigma} \leq c^{\sigma}\left(1+K^{-\sigma}\right)^{-1}\left(\langle\eta\rangle^{\sigma}+\langle\xi-\eta\rangle^{\sigma}\right) .
$$

Thus we are done if 2.10 holds with

$$
1<c<\left(1+K^{-\sigma}\right)^{1 / \sigma} .
$$

Otherwise, there holds

$$
|\xi| \geq\left(1+K^{-\sigma}\right)^{1 / \sigma}|\xi-\eta|=: \widetilde{c}|\xi-\eta|,
$$

and since $\widetilde{c}>1$, we may then apply 2.7 . This yields

$$
\langle\xi\rangle^{\sigma} \leq\langle\eta\rangle^{\sigma}+\left(\widetilde{c}^{\sigma}-(\widetilde{c}-1)^{\sigma}\right)\langle\xi-\eta\rangle^{\sigma}
$$

and the result follows. The proof of $(2.9)$ is trivial, hence omitted.

Remark 2. Inequality 2.7) is somehow similar to inequality (3.11) in [BMM13, which we reproduce here:

$$
\left|\langle\xi\rangle^{\sigma}-\langle\eta\rangle^{\sigma}\right| \leq \frac{\sigma}{(K-1)^{1-\sigma}}\langle\xi-\eta\rangle^{\sigma}
$$

Note that the coefficient $\frac{\sigma}{(K-1)^{1-\sigma}}$ may be strictly greater than 1 . Inequality 2.8 is similar to inequality (3.12) in [BMM13], which we reproduce here:

$$
\langle\xi\rangle^{\sigma} \leq\left(\frac{\langle\eta\rangle^{\sigma}}{\langle\xi\rangle^{\sigma}}\right)^{1-\sigma}\left(\langle\eta\rangle^{\sigma}+\langle\xi-\eta\rangle^{\sigma}\right)
$$

for $|\eta| \geq|\xi-\eta|$. Again, the coefficient $\left(\frac{\langle\eta\rangle^{\sigma}}{\langle\xi\rangle^{\sigma}}\right)^{1-\sigma}$ may be strictly greater than 1 . 


\subsection{Classes of symbols}

We define a class of symbols $a(x, \xi)$ with Gevrey regularity in the spatial variable $x$.

Definition 2.3 (Class of symbols with Gevrey regularity). For $s \in(1, \infty)$ and $R>0$, for $m \in \mathbb{R}, \rho$ and $\delta$ such that $0<\delta<\rho \leq 1$, we define $\mathrm{S}_{\rho, \delta}^{m} \mathrm{G}_{R}^{s}$ to be the class of symbols $a(x, \xi)$ for which there is a bounded sequence of positive numbers $C_{\alpha, \beta}$ such that

$$
\left|\partial_{x}^{\alpha} \partial_{\xi}^{\beta} a(x, \xi)\right| \leq C_{\alpha, \beta} R^{|\alpha+\beta|}|\alpha| !^{s}|\beta| !\langle\xi\rangle^{m-\rho|\beta|+\delta|\alpha|}
$$

uniformly in $x \in \mathbb{R}^{d}$ and $\xi \in \mathbb{R}^{d}$. We denote

$$
|a|_{\alpha, \beta}=\sup _{(x, \xi) \in \mathbb{R}^{d} \times \mathbb{R}^{d}}\left|R^{-|\alpha+\beta|}\right| \alpha\left|!^{-s}\right| \beta\left|!^{-1}\langle\xi\rangle^{-m+\rho|\beta|-\delta|\alpha|} \partial_{x}^{\alpha} \partial_{\xi}^{\beta} a(x, \xi)\right| .
$$

Remark 3. Note that the space $G_{R}^{s}$ is naturally in $\mathrm{S}_{0,0}^{0} \mathrm{G}_{R}^{s}$, with

$$
|a|_{\alpha, \beta} \leq|a|_{s, R} \quad, \quad \forall(\alpha, \beta) \in \mathbb{Z}^{d} \times \mathbb{Z}^{d} .
$$

Moreover spaces $\mathrm{S}_{\rho, \delta}^{m} \mathrm{G}_{R}^{s}$ are naturally embedded in $S_{\rho, \delta}^{m}$.

Remark 4. A way to look at inequalities 2.11) is to put together the Gevrey term $R^{|\alpha|}|\alpha| !^{s}$ and the typical pseudo-differential term $\langle\xi\rangle^{\delta|\alpha|}$, which means that $a(\cdot, \xi)$ is in $G_{R\langle\xi\rangle^{\delta}}^{\text {for }}$ all $\xi \in \mathbb{R}^{d}$ : the Gevrey radius in $x$ of the symbol decreases with $|\xi|$ if $\delta>0$.

\section{Conjugation of a Gevrey function}

We consider the Gevrey conjugation operator of a function $F$ in $\mathcal{G}_{\tau}^{\sigma}$ with $\tau \geq 0$, and we denote

$$
F^{(\tau)}=e^{\tau D^{\sigma}} F e^{-\tau D^{\sigma}}
$$

where $D=\mathrm{op}(\langle\cdot\rangle)$.

Proposition 3.1. Assume that $D^{m} F \in \mathcal{G}_{\tau}^{\sigma}$ for some $m \geq 0$. Then, for any $v \in H^{m}$, there holds

$$
\left|F^{(\tau)} v\right|_{H^{m}} \lesssim\left|D^{m} F\right|_{\sigma, \tau}|v|_{L^{2}}+|F|_{\sigma, \tau}|v|_{H^{m}} .
$$

Remark 5. This implies in particular that $H^{m} \mathcal{G}_{\tau}^{\sigma}$, the space of Gevrey functions with Sobolev correction of order $m$, is an algebra for any $m \geq 0$. With $m=0$ in proposition 3.1. we see that if $F$ is in $\mathcal{G}_{\tau}^{\sigma}$, then $F^{(\tau)}$ operates in $L^{2}$.

Proof. In Fourier there holds

$$
\mathcal{F}\left(D^{m} F^{(\tau)} v\right)(\xi)=\int_{\eta} e^{\tau\langle\xi\rangle^{\sigma}-\tau\langle\eta\rangle^{\sigma}}\langle\xi\rangle^{m} \widehat{F}(\xi-\eta) \widehat{v}(\eta) d \eta .
$$

We use here a paraproduct decomposition and Lemma 2.2. Let $K>1$. We divide the integral in $\eta \in \mathbb{R}^{d}$ in three frequency regions, defined as $\mathcal{R}_{1}=\left\{\eta:|\xi-\eta| \leq \frac{1}{K}|\eta|\right\}$, 
$\mathcal{R}_{2}=\left\{\eta:|\eta| \leq \frac{1}{K}|\xi-\eta|\right\}$ and $\mathcal{R}_{3}=\left\{\eta: \frac{1}{K}|\xi-\eta|<|\eta|<K|\xi-\eta|\right\}$. We consider then each region successively:

- The case where $|\xi-\eta| \leq \frac{1}{K}|\eta|$ : thanks to inequality 2.7) in Lemma 2.2 there is $c \in(0,1)$ such that $\langle\xi\rangle^{\sigma}-\langle\eta\rangle^{\sigma} \leq c\langle\xi-\eta\rangle^{\sigma}$, hence

$$
e^{\tau\langle\xi\rangle^{\sigma}-\tau\langle\eta\rangle^{\sigma}} \leq e^{c \tau\langle\xi-\eta\rangle^{\sigma}} .
$$

Besides, in the region under consideration, the Sobolev term satisfies $\langle\xi\rangle^{m} \lesssim\langle\eta\rangle^{m}$. This implies that

$$
\begin{aligned}
& \int_{\eta \in \mathcal{R}_{1}} e^{\tau\langle\xi\rangle^{\sigma}-\tau\langle\eta\rangle^{\sigma}}\langle\xi\rangle^{m}|\widehat{F}(\xi-\eta)||\widehat{v}(\eta)| d \eta \\
& \lesssim \int_{\eta \in \mathcal{R}_{1}} e^{c \tau\langle\xi-\eta\rangle^{\sigma}}\langle\eta\rangle^{m}|\widehat{F}(\xi-\eta)||\widehat{v}(\eta)| d \eta \\
& \lesssim \int_{\eta \in \mathcal{R}_{1}} e^{-\tau(1-c)\langle\xi-\eta\rangle^{\sigma}} e^{\tau\langle\xi-\eta\rangle^{\sigma}}|\widehat{F}(\xi-\eta)|\langle\eta\rangle^{m}|\widehat{v}(\eta)| d \eta .
\end{aligned}
$$

We use next Young's inequality to obtain

$$
\begin{aligned}
& \left|\int_{\eta \in \mathcal{R}_{1}} e^{-\tau(1-c)\langle\xi-\eta\rangle^{\sigma}} e^{\tau\langle\xi-\eta\rangle^{\sigma}}\right| \widehat{F}(\xi-\eta)\left|\langle\eta\rangle^{m}\right| \widehat{v}(\eta)|d \eta|_{L_{\xi}^{2}} \\
& \lesssim\left|e^{-\tau(1-c)\langle\cdot\rangle^{\sigma}} e^{\tau\langle\cdot\rangle^{\sigma}}\right| \widehat{F}(\cdot)||_{L^{1}}|v|_{H^{m}} \\
& \lesssim\left|e^{-\tau(1-c)\langle\cdot\rangle^{\sigma}}\right|_{L^{2}}|F|_{\sigma, \tau}|v|_{H^{m}}
\end{aligned}
$$

using the extra Gevrey weight $e^{-\tau(1-c)\langle\xi-\eta\rangle^{\sigma}}$.

- The case where $|\eta| \leq \frac{1}{K}|\xi-\eta|$ : thanks to inequality 2.7] in Lemma 2.2, there is $c \in(0,1)$ such that $\langle\xi\rangle^{\sigma}-\langle\xi-\eta\rangle^{\sigma} \leq c\langle\eta\rangle^{\sigma}$, hence

$$
e^{\langle\xi\rangle^{\sigma}-\langle\xi-\eta\rangle^{\sigma}} \leq e^{c\langle\eta\rangle^{\sigma}}
$$

Besides, in the region under consideration, the Sobolev term satisfies $\langle\xi\rangle^{m} \lesssim\langle\xi-\eta\rangle^{m}$. This implies that

$$
\begin{aligned}
& \int_{\eta \in \mathcal{R}_{2}} e^{\tau\langle\xi\rangle^{\sigma}-\tau\langle\eta\rangle^{\sigma}}\langle\xi\rangle^{m}|\widehat{F}(\xi-\eta)| \widehat{v}(\eta) \mid d \eta \\
& \lesssim \int_{\eta \in \mathcal{R}_{2}} e^{\tau\langle\xi\rangle^{\sigma}-\tau\langle\eta\rangle^{\sigma}-\tau\langle\xi-\eta\rangle^{\sigma}}\langle\xi-\eta\rangle^{m} e^{\tau\langle\xi-\eta\rangle^{\sigma}}|\widehat{F}(\xi-\eta)||\widehat{v}(\eta)| d \eta \\
& \lesssim \int_{\eta \in \mathcal{R}_{2}} e^{-\tau(1-c)\langle\eta\rangle^{\sigma}}\langle\xi-\eta\rangle^{m} e^{\tau\langle\xi-\eta\rangle^{\sigma}}|\widehat{F}(\xi-\eta)||\widehat{v}(\eta)| d \eta
\end{aligned}
$$

We use next Young's inequality to obtain

$$
\begin{aligned}
& \left|\int_{\eta \in \mathcal{R}_{2}} e^{-\tau(1-c)\langle\eta\rangle^{\sigma}}\langle\xi-\eta\rangle^{m} e^{\tau\langle\xi-\eta\rangle^{\sigma}}\right| \widehat{F}(\xi-\eta)|| \widehat{v}(\eta)|d \eta|_{L_{\xi}^{2}} \\
& \lesssim\left|e^{-\tau(1-c)\langle\cdot\rangle^{\sigma}}\right| \widehat{v}(\eta)||_{L^{1}}\left|D^{m} F\right|_{\sigma, \tau} \\
& \lesssim\left|e^{-\tau(1-c)\langle\cdot\rangle^{\sigma}}\right|_{L^{2}}\left|D^{m} F\right|_{\sigma, \tau}|v|_{L^{2}}
\end{aligned}
$$


using the extra Gevrey weight $e^{-\tau(1-c)\langle\xi-\eta\rangle^{\sigma}}$.

- The case where $\frac{1}{K}|\xi-\eta|<|\eta|<K|\xi-\eta|$ : thanks to inequality (2.8) in Lemma 2.2. there is $c^{\prime} \in(0,1)$ such that $\langle\xi\rangle^{\sigma} \leq c^{\prime}\langle\xi-\eta\rangle^{\sigma}+\langle\eta\rangle^{\sigma}$, hence

$$
e^{\tau\langle\xi\rangle^{\sigma}-\tau\langle\eta\rangle^{\sigma}} \leq e^{c^{\prime}\langle\xi-\eta\rangle^{\sigma}} .
$$

Besides, in the region under consideration, the Sobolev term satisfies $\langle\xi\rangle^{m} \lesssim\langle\eta\rangle^{m}+\langle\xi-\eta\rangle^{m}$ where the implicit constant depends on $m$, thus

$$
\begin{aligned}
& \int_{\eta \in \mathcal{R}_{3}} e^{\tau\langle\xi\rangle^{\sigma}-\tau\langle\eta\rangle^{\sigma}}\langle\xi\rangle^{m}|\widehat{F}(\xi-\eta)||\widehat{v}(\eta)| d \eta \\
& \lesssim \int_{\eta \in \mathcal{R}_{3}} e^{-\left(1-c^{\prime}\right) \tau\langle\xi-\eta\rangle^{\sigma}}\left(\langle\eta\rangle^{m}+\langle\xi-\eta\rangle^{m}\right) e^{\tau\langle\xi-\eta\rangle^{\sigma}}|\widehat{F}(\xi-\eta)||\widehat{v}(\eta)| d \eta \\
& \lesssim \int_{\eta \in \mathcal{R}_{3}} e^{-\left(1-c^{\prime}\right) \tau\langle\xi-\eta\rangle^{\sigma}} e^{\tau\langle\xi-\eta\rangle^{\sigma}}|\widehat{F}(\xi-\eta)|\langle\eta\rangle^{m}|\widehat{v}(\eta)| d \eta \\
& \quad+\int_{\eta \in \mathcal{R}_{3}} e^{-\left(1-c^{\prime}\right) \tau\langle\xi-\eta\rangle^{\sigma}}\langle\xi-\eta\rangle^{m} e^{\tau\langle\xi-\eta\rangle^{\sigma}}|\widehat{F}(\xi-\eta)||\widehat{v}(\eta)| d \eta
\end{aligned}
$$

We use next Young's inequality to obtain

$$
\begin{aligned}
& \left|\int_{\eta \in \mathcal{R}_{3}} e^{\tau\langle\xi\rangle^{\sigma}-\tau\langle\eta\rangle^{\sigma}}\langle\xi\rangle^{m}\right| \widehat{F}(\xi-\eta)|| \widehat{v}(\eta)|d \eta|_{L_{\xi}^{2}} \\
& \lesssim\left|e^{-\left(1-c^{\prime}\right) \tau\langle\cdot\rangle^{\sigma}}\right|_{L^{2}}\left(\left|D^{m} F\right|_{\sigma, \tau}|v|_{L^{2}}+|F|_{\sigma, \tau}|v|_{H^{m}}\right)
\end{aligned}
$$

The result follows from $\left(3.2\right.$, viewed as an integral over $\mathcal{R}_{1} \cup \mathcal{R}_{2} \cup \mathcal{R}_{3}$.

\section{Action of pseudo-differential operators on Gevrey spaces}

In this Section, we consider symbols in $\mathrm{S}_{\rho, \delta}^{0} \mathrm{G}_{R}^{s}$ with compact support $B$ of $\mathbb{R}_{x}^{d}$, uniformly in $\xi \in \mathbb{R}^{d}$. This additional assumption on the support of the symbol allows to use Proposition 2.1. parlaying the spatial Gevrey regularity into a Fourier Gevrey regularity for $a(\cdot, \xi)$. We may then use an adapted paraproduct decomposition to prove the continuous action of operators with symbols in $\mathrm{S}_{\rho, \delta}^{0} \mathrm{G}_{R}^{s}$.

In all the following we consider quantizations of the type

$$
\mathrm{op}_{h}(a) u(x)=\int e^{i(x-y) \cdot \eta} a(x-h(x-y), \eta) u(y) d y d \eta .
$$

with $h \in[0,1]$. First we prove this result in the particular case $\rho=1, \delta=0$.

Theorem 4.1 (Action of $S_{1,0}^{0} G_{R}^{s}$ on $\mathcal{G}_{\tau}^{\sigma}$ ). Let $s \in(1, \infty)$ and $R>0$. Let a be in $\mathrm{S}_{1,0}^{0} \mathrm{G}_{R}^{s}$, constant outside a compact set $B$ of $\mathbb{R}_{x}^{d}$, uniformly in $\xi \in \mathbb{R}^{d}$. Then for any $\tau<s R^{-1 / s}$ and $\sigma=\frac{1}{s}$, the operator $\operatorname{op}_{h}(a)$ acts continuously on $\mathcal{G}_{\tau}^{\sigma}$ with norm

$$
\left\|\operatorname{op}_{h}(a)\right\|_{\mathcal{L}\left(\mathcal{G}_{\tau}^{\sigma}\right)} \lesssim_{h}|B|^{1 / 2} C\left(\tau s^{-1} R^{1 / s}\right) \sup _{\alpha \in \mathbb{N}^{d}}|a|_{\alpha, 0}
$$

where $C\left(\tau s^{-1} R^{1 / s}\right)$ is defined in 2.6$)$. 
Proof. First, for fixed $\xi \in \mathbb{R}^{d}$, as $a(\cdot, \xi)$ is in $\mathrm{G}_{R}^{s}$ with compact support, Proposition 2.1 implies that $\widehat{a}(\cdot, \xi)$, the Fourier transform with respect to $x$ of $a(\cdot, \xi)$, is in $\mathcal{G}_{\tau}^{\sigma}$ uniformly in $\xi \in \mathbb{R}^{d}$, with $\sigma=1 / s$ and $\tau<s R^{-1 / s}$. That is, we may write

$$
\widehat{a}(\zeta, \xi)=F_{\xi}(\zeta)
$$

where for fixed $\xi \in \mathbb{R}^{d}, F_{\xi}(\cdot)$ belongs to $\mathcal{G}_{\tau}^{\sigma}$ with the uniform (in $\xi$ ) bound

$$
\left|F_{\xi}(\cdot)\right|_{\sigma, \tau} \leq|B|^{1 / 2} C\left(\tau s^{-1} R^{1 / s}\right)|a(\cdot, \xi)|_{s, R} .
$$

thanks to Proposition 2.1. By definition 2.12 of the semi-norms in Definition 2.3, there holds

$$
\left|F_{\xi}(\cdot)\right|_{\sigma, \tau} \leq|B|^{1 / 2} C\left(\tau s^{-1} R^{1 / s}\right) \sup _{\alpha \in \mathbb{N}^{d}}|a|_{\alpha, 0} .
$$

Let $u$ be in $G_{\tau}^{\sigma}$, and denote $v(\eta)=e^{\tau\langle\eta\rangle^{\sigma}} \hat{u}(\eta)$ which is in $L^{2}$. We compute the Fourier transform of $\operatorname{op}_{h}(p) u$. The case $h=0$ is simple, as the Fourier transform of $\mathrm{op}_{0}(p) u$ is

$$
\int_{\eta} \widehat{a}(\xi-\eta, \xi) \hat{u}(\eta) d \eta
$$

Thus there holds

$$
e^{\tau\langle\xi\rangle^{\sigma}} \mathcal{F}\left(\mathrm{op}_{0}(a) u\right)(\xi)=\int_{\eta} e^{\tau\langle\xi\rangle^{\sigma}-\tau\langle\eta\rangle^{\sigma}} F_{\xi}(\xi-\eta) v(\eta) d \eta .
$$

Proposition 3.1 now yields the result for $h=0$, since the bound 4.2 is uniform in $\xi$.

For $h \in(0,1]$, the computation of the Fourier transform is more delicate, and there holds

$$
\begin{aligned}
\mathcal{F}\left(\operatorname{op}_{h}(a) u\right)(\xi) & =\int e^{-i x \cdot \xi} e^{i(x-y) \cdot \eta} a(x-h(x-y), \eta) u(y) d y d \eta d x \\
& =\int e^{-i x \cdot \xi} e^{i(x-y) \cdot \eta} e^{i y \cdot \zeta} a(x-h(x-y), \eta) \widehat{u}(\zeta) d \zeta d y d \eta d x
\end{aligned}
$$

We define new variables, putting

$$
\widetilde{x}=(1-h) x+h y \quad, \quad \widetilde{y}=(1-h) x-h y
$$

which leads to

$$
\begin{aligned}
& \int e^{-i x \cdot \xi} e^{i(x-y) \cdot \eta} e^{i y \cdot \zeta} a(x-h(x-y), \eta) \widehat{u}(\zeta) d \zeta d y d \eta d x \\
& =\int e^{i \widetilde{y} \cdot \varphi_{1}(\xi, \zeta, \eta)} e^{i \widetilde{x} \cdot \varphi_{2}(\xi, \zeta, \eta)} a(\widetilde{x}, \eta) \widehat{u}(\zeta) d \zeta d \widetilde{y} d \eta d \widetilde{x}
\end{aligned}
$$

with

$$
\varphi_{1}(\xi, \zeta, \eta)=-\frac{1}{2(1-h)} \xi-\frac{1}{2 h} \zeta+\frac{1}{2 h(1-h)} \eta
$$


and

$$
\varphi_{2}(\xi, \zeta, \eta)=-\frac{1}{2(1-h)} \xi+\frac{1}{2 h} \zeta+\frac{2 h-1}{2 h(1-h)} \eta .
$$

As the integrand depends on $\tilde{y}$ only through the phase term, there holds

$$
\begin{aligned}
& \int e^{i \widetilde{y} \cdot \varphi_{1}(\xi, \zeta, \eta)} e^{i \widetilde{x} \cdot \varphi_{2}(\xi, \zeta, \eta)} a(\widetilde{x}, \eta) \widehat{u}(\zeta) d \zeta d \widetilde{y} d \eta d \widetilde{x} \\
& =\int_{\left\{(\zeta, \eta): \varphi_{1}(\xi, \zeta, \eta)=0\right\}} e^{i \widetilde{x} \cdot \varphi_{2}(\xi, \zeta, \eta)} a(\widetilde{x}, \eta) \widehat{u}(\zeta) d \zeta d \eta d \widetilde{x} \\
& =\int_{\left\{(\zeta, \eta): \varphi_{1}(\xi, \zeta, \eta)=0\right\}} e^{i \widetilde{x} \cdot(\zeta-\eta) / h} a(\widetilde{x}, \eta) \widehat{u}(\zeta) d \zeta d \eta d \widetilde{x}
\end{aligned}
$$

by definition 4.4 of $\varphi_{2}$. Hence finally

$$
\mathcal{F}\left(\operatorname{op}_{h}(a) u\right)(\xi)=\int_{\left\{(\zeta, \eta): \varphi_{1}(\xi, \zeta, \eta)=0\right\}} \widehat{a}((\zeta-\eta) / h, \eta) \widehat{u}(\zeta) d \zeta d \eta
$$

As in the proof for $h=0$, we write

$$
\begin{aligned}
& e^{\tau\langle\xi\rangle^{\sigma}} \mathcal{F}\left(\mathrm{op}_{h}(a) u\right)(\xi) \\
& =\int_{\left\{(\zeta, \eta): \varphi_{1}(\xi, \zeta, \eta)=0\right\}} e^{\tau\langle\xi\rangle^{\sigma}-\tau\langle\zeta\rangle^{\sigma}} \widehat{a}((\zeta-\eta) / h, \eta) v(\zeta) d \zeta d \eta
\end{aligned}
$$

On the surface $\left\{(\zeta, \eta): \varphi_{1}(\xi, \zeta, \eta)=0\right\}$ there holds

$$
\xi=-\frac{1-h}{h} \zeta+\frac{1}{h} \eta=\frac{1}{h}(\eta-\zeta)+\zeta
$$

hence

$$
e^{\tau\langle\xi\rangle^{\sigma}} \mathcal{F}\left(\operatorname{op}_{h}(a) u\right)(\xi)=\int_{\left\{(\zeta, \eta): \varphi_{1}(\xi, \zeta, \eta)=0\right\}} e^{\tau\langle\xi\rangle^{\sigma}-\tau\langle\zeta\rangle^{\sigma}} F_{\eta}(\xi-\zeta) v(\zeta) d \zeta d \eta
$$

with $F$ defined in 4.1). We may then conclude in the same as the case $h=0$.

In the general case $0<\delta<\rho \leq 1$, Remark 4 indicates a potential obstruction for the Gevrey index. This is made precise in the following

Theorem 4.2 (Action of $S_{\rho, \delta}^{0} G_{R}^{s}$ on $\mathcal{G}_{\tau}^{\sigma}$ ). Let $s \in(1, \infty), R>0$ and $0<\delta<\rho \leq 1$. Let a be in $\mathrm{S}_{\rho, \delta}^{0} \mathrm{G}_{R}^{s}$, constant outside a compact set $B$ of $\mathbb{R}_{x}^{d}$, uniformly in $\xi \in \mathbb{R}^{d}$. Then for any

$$
\sigma \leq(1-\delta) / s \quad \text { and } \quad \tau^{\prime}<\tau<s R^{-1 / s}
$$

the operator $\operatorname{op}(a)$ acts continuously from $\mathcal{G}_{\tau}^{\sigma}$ into $\mathcal{G}_{\tau^{\prime}}^{\sigma}$ with norm

$$
\left\|\operatorname{op}_{0}(a)\right\|_{\mathcal{L}\left(\mathcal{G}_{\tau}^{\sigma}, \mathcal{G}_{\tau^{\prime}}^{\sigma}\right)} \lesssim|B|^{1 / 2} C\left(\tau s^{-1} R^{1 / s}\right) \sup _{\alpha \in \mathbb{N}^{d}}|a|_{\alpha, 0}
$$

where $C\left(\tau s^{-1} R^{1 / s}\right)$ is defined in 2.6 . 
Proof. First, for fixed $\xi \in \mathbb{R}^{d}$, as $a(\cdot, \xi)$ is in $\mathrm{G}_{R}^{s}$ with compact support, Proposition 2.1 and Remark 4 implies that $\widehat{a}(\cdot, \xi)$, the Fourier transform with respect to $x$ of $a(\cdot, \xi)$, is in $\mathcal{G}_{\tau\langle\xi\rangle^{-\delta / s}}^{\sigma}$ uniformly in $\xi \in \mathbb{R}^{d}$, with $\sigma=1 / s$ and $\tau<s R^{-1 / s}$. That is, we may write

$$
e^{\tau\langle\xi\rangle^{-\delta / s}\langle\zeta\rangle^{1 / s}} \hat{a}(\zeta, \xi)=F_{\xi}(\zeta)
$$

where for fixed $\xi \in \mathbb{R}^{d}, F_{\xi}(\cdot)$ belongs to $L^{2}$ with the uniform (in $\xi$ ) bound

$$
\left|F_{\xi}(\cdot)\right|_{L^{2}} \leq|B|^{1 / 2} C\left(\tau s^{-1} R^{1 / s}\right)|a(\cdot, \xi)|_{s, R}
$$

thanks to Proposition 2.1. By definitions 2.12) of the semi-norms in Definition 2.3, there holds

$$
\left|F_{\xi}(\cdot)\right|_{\sigma, \tau} \leq|B|^{1 / 2} C\left(\tau s^{-1} R^{1 / s}\right) \sup _{\alpha \in \mathbb{N}^{d}}|a|_{\alpha, 0} .
$$

Let $u$ be in $\mathcal{G}_{\tau}^{\sigma}$. Denoting $v(\eta)=e^{\tau\langle\eta\rangle^{\sigma}} \hat{u}(\eta)$, there holds

$$
e^{\tau^{\prime}\langle\xi\rangle^{\sigma}} \mathcal{F}\left(\operatorname{op}_{0}(a) u\right)(\xi)=\int_{\eta} e^{\tau^{\prime}\langle\xi\rangle^{\sigma}-\tau\langle\eta\rangle^{\sigma}-\tau\langle\xi\rangle^{-\delta / s}\langle\xi-\eta\rangle^{1 / s}} F_{\xi}(\xi-\eta) v(\eta) d \eta
$$

We now decompose the integral into three regions, as in the proof of Proposition 3.1 Once we derive appropriate bounds on the exponential factor

$$
W\left(\tau^{\prime} ; \tau\right):=\exp \left(\tau^{\prime}\langle\xi\rangle^{\sigma}-\tau\langle\eta\rangle^{\sigma}-\tau\langle\xi\rangle^{-\delta / s}\langle\xi-\eta\rangle^{1 / s}\right)
$$

the result follows from 4.5 by application of Young's inequality, as in the proof of Proposition 3.1. Thus we focus only on the above exponential factor. Here the multiplicative coefficient $K>1$ is chosen in terms of $\tau$ and $\tau^{\prime}$.

- The case where $|\xi-\eta| \leq \frac{1}{K}|\eta|$ : here the weight $\langle\xi\rangle^{-\delta / s}$ in the Gevrey radius of $\hat{a}(\cdot, \xi)$ is small, and

$$
W\left(\tau^{\prime} ; \tau\right) \leq \exp \left(\tau^{\prime}\langle\xi\rangle^{\sigma}-\tau\langle\eta\rangle^{\sigma}\right) .
$$

With (2.7) there holds

$$
W\left(\tau^{\prime} ; \tau\right) \leq \exp \left(\tau^{\prime}\left(K^{\sigma}-(K-1)^{\sigma}\right)\langle\xi-\eta\rangle^{\sigma}-\left(\tau-\tau^{\prime}\right)\langle\eta\rangle^{\sigma}\right)
$$

Using now $|\xi-\eta| \leq \frac{1}{K}|\eta|$, there holds $\langle\xi-\eta\rangle^{\sigma} \leq\langle\eta\rangle^{\sigma}$, so that

$$
W\left(\tau^{\prime} ; \tau\right) \leq \exp \left(-\left(\tau-\left(1+\left(K^{\sigma}-(K-1)^{\sigma}\right)\right) \tau^{\prime}\right)\langle\eta\rangle^{\sigma}\right) .
$$

For $K$ large enough, depending only on $\tau^{\prime}$ and $\tau$, with $\tau^{\prime}<\tau$, there holds $\tau-(1+$ $\left.\left(K^{\sigma}-(K-1)^{\sigma}\right)\right) \tau^{\prime}$, thus $|W|_{L_{\eta}^{2}}<\infty$.

- The case where $|\eta| \leq \frac{1}{K}|\xi-\eta|$ : since $|\eta| \leq K^{-1}|\xi-\eta|$, there holds $|\xi| \leq\left(1+K^{-1}\right) \mid \xi-$ $\eta \mid$, hence $\langle\xi\rangle \leq\left(1+K^{-1}\right)\langle\xi-\eta\rangle$. Thus, with $\sigma \leq(1-\delta) / s$, we find the bound

$$
W\left(\tau^{\prime} ; \tau\right) \leq \exp \left(\tau^{\prime}\langle\xi\rangle^{\sigma}-\tau\langle\eta\rangle^{\sigma}-\tau(1+1 / K)^{-\delta / s}\langle\xi-\eta\rangle^{(1-\delta) / s}\right) .
$$


Using inequality (2.7), this implies

$$
\begin{aligned}
W\left(\tau^{\prime} ; \tau\right) \leq \exp \left(-\left(\tau-\left(K^{\sigma}-(K-1)^{\sigma}\right) \tau^{\prime}\right)\langle\eta\rangle^{\sigma}\right) \\
\quad \times \exp \left(-\left(\tau(1+1 / K)^{-\delta / s}-\tau^{\prime}\right)\langle\xi-\eta\rangle^{(1-\delta) / s}\right)
\end{aligned}
$$

Since $K<1$ and $\tau>\tau^{\prime}$, there holds $\tau-\left(K^{\sigma}-(K-1)^{\sigma}\right) \tau^{\prime}>0$. Thus

$$
W\left(\tau^{\prime} ; \tau\right) \leq \exp \left(-\left(\tau(1+1 / K)^{-\delta / s}-\tau^{\prime}\right)\langle\xi-\eta\rangle^{(1-\delta) / s}\right)
$$

and if $K$ is large enough, depending only on $\tau$ and $\tau^{\prime}$, there holds $\tau(1+1 / K)^{-\delta / s}-\tau^{\prime}>0$. Thus $|W|_{L_{\eta}^{2}}<\infty$.

- The case where $\frac{1}{K}|\xi-\eta|<|\eta|<K|\xi-\eta|$ : here we use inequality (2.8), which implies, since $\sigma \leq(1-\delta) / s$,

$$
W\left(\tau^{\prime} ; \tau\right) \leq \exp \left(-\left(\tau-\tau^{\prime}\right)\langle\eta\rangle^{\sigma}\right) \exp \left(-\left(\tau\langle\xi\rangle^{-\delta / s}-\tau^{\prime}\langle\xi-\eta\rangle^{-\delta / s}\right)\langle\xi-\eta\rangle^{1 / s}\right),
$$

where $c^{\prime}=c^{\prime}(K) \in(0,1)$. Since $|\xi| \leq(1+K)|\xi-\eta|$ in the region under consideration, hence $\langle\xi\rangle \leq(1=K)\langle\xi-\eta\rangle$, this implies

$$
W \leq \exp \left(-\left(\tau(1+K)^{-\delta / s}-\tau^{\prime}\right)\langle\xi-\eta\rangle^{(1-\delta) / s}\right)
$$

thus if $K$ is large enough, depending only on $\tau$ and $\tau^{\prime}$, there holds $|W|_{L_{\eta}^{2}}<\infty$.

\section{A conjugation Lemma for operators}

We consider here a symbol $a$ in $\mathrm{S}_{\rho, 0}^{m} \mathrm{G}_{R}^{s}$ for $\rho \in[0,1], R>0$ and $s \in(1, \infty)$, with compact support $B$ of $\mathbb{R}_{x}^{d}$, uniformly in $\xi \in \mathbb{R}^{d}$. It is known (see Lemma 7.1 in [CNR]) that there is a symbol $\widetilde{a}$ such that

$$
\operatorname{op}(\widetilde{a})=\operatorname{op}(a)^{(\tau)}=e^{\tau D^{\sigma}} \operatorname{op}(a) e^{-\tau D^{\sigma}}
$$

and which satisfies

$$
\widetilde{a}(x, \xi)=\int_{y, \eta} e^{-i \eta \cdot y} e^{\tau\langle\xi+\eta\rangle^{\sigma}-\tau\langle\xi\rangle^{\sigma}} a(x+y, \xi) d y d \eta .
$$

In Proposition 2.1 in $\left[\mathrm{CNR}\right.$, the symbol $\widetilde{a}$ is proved to be in $S_{1,0}^{m}$ for small $\tau$. We extend here the result for all $|\tau|<s R^{-1 / s}$, with in addition an estimate of the semi-norms of the symbol.

Lemma 5.1. Given a in $\mathrm{S}_{\rho, 0}^{m} \mathrm{G}_{R}^{s}$, for any $|\tau|<s R^{-1 / s}$, the symbol defined by 5.2 is in $\mathrm{S}_{1,0}^{m}$. Moreover, for any $\underline{\tau} \in\left(|\tau|, s R^{-1 / s}\right)$, for any $\alpha, \beta$ in $\mathbb{N}^{d}$ there holds

$$
\sup _{x \in B, \xi \in \mathbb{R}^{d}}\left|\langle\xi\rangle^{-m+|\beta|} \partial_{x}^{\alpha} \partial_{\xi}^{\beta} \widetilde{a}(x, \xi)\right| \lesssim|B|^{1 / 2} C\left(\underline{\tau} s^{-1} R^{1 / s}\right) \sup _{\alpha \in \mathbb{N}^{d}}|a|_{\alpha, \beta}(\underline{\tau}-|\tau|)^{-(2|\beta|+|\alpha|) / \sigma}
$$

where constant $C$ is defined in 2.6. 
Proof. We compute the derivatives of the symbol $\widetilde{a}$. There holds

$$
\begin{aligned}
& \partial_{x}^{\alpha} \partial_{\xi}^{\beta} \widetilde{a}(x, \xi) \\
& =\sum_{\beta_{1}+\beta_{2}=\beta}\left(\begin{array}{c}
\beta \\
\beta_{1}, \beta_{2}
\end{array}\right) \int_{y, \eta} e^{-i \eta \cdot y} \partial_{\xi}^{\beta_{1}}\left(e^{\tau\langle\xi+\eta\rangle^{\sigma}-\tau\langle\xi\rangle^{\sigma}}\right) \partial_{x}^{\alpha} \partial_{\xi}^{\beta_{2}} a(x+y, \xi) d y d \eta \\
& =\sum_{\beta_{1}+\beta_{2}=\beta}\left(\begin{array}{c}
\beta \\
\beta_{1}, \beta_{2}
\end{array}\right) \int_{\eta} e^{i \eta \cdot x} \partial_{\xi}^{\beta_{1}}\left(e^{\tau\langle\xi+\eta\rangle^{\sigma}-\tau\langle\xi\rangle^{\sigma}}\right)(i \eta)^{\alpha} \partial_{\xi}^{\beta_{2}} \hat{a}(\eta, \xi) d \eta .
\end{aligned}
$$

We use now the fact that $a$ is in $\mathrm{S}_{\rho, 0}^{m} \mathrm{G}_{R}^{s}$ with compact support $B$ in $\mathbb{R}_{x}^{d}$, uniformly in $\xi$. Thanks to Proposition 2.1, we may write

$$
e^{\underline{\tau}\langle\eta\rangle^{\sigma}}\langle\xi\rangle^{-m+\rho\left|\beta_{2}\right|} \partial_{\xi}^{\beta_{2}} \hat{a}(\eta, \xi)=F_{\xi, \beta_{2}}(\eta),
$$

where for fixed $\xi \in \mathbb{R}^{d}$ and $\beta_{2} \in \mathbb{N}^{d}, F_{\xi, \beta_{2}}$ is in $L_{\eta}^{2}$ with bound

$$
\left|F_{\xi, \beta_{2}}\right|_{L_{\eta}^{2}} \lesssim|B|^{1 / 2} C\left(\underline{\tau} s^{-1} R^{1 / s}\right) \sup _{\alpha \in \mathbb{N}^{d}}|a|_{\alpha, \beta_{2}}
$$

uniformly in $\xi \in \mathbb{R}^{d}$ and $\beta_{2} \in \mathbb{N}^{d}$, and for all $\underline{\tau}<s R^{-1 / s}$. The semi-norms of $a$ are defined in 2.12). Next, as proved in the course of Proposition 2.1 in [CNR], there holds

$$
\left|\partial_{\xi}^{\beta_{1}}\left(e^{\tau\langle\xi+\eta\rangle^{\sigma}-\tau\langle\xi\rangle^{\sigma}}\right)\right| \lesssim\langle\xi\rangle^{-\left|\beta_{1}\right|}\langle\eta\rangle^{2\left|\beta_{1}\right|} e^{\tau\langle\xi+\eta\rangle^{\sigma}-\tau\langle\xi\rangle^{\sigma}}
$$

This is proved using Faà di Bruno formula (see Lemma 5.1 in [Mor17a]) and inequality $\partial_{\xi}^{\beta_{1}}\left(\langle\xi+\eta\rangle^{\sigma}-\langle\xi\rangle^{\sigma}\right) \lesssim\langle\xi\rangle^{-\left|\beta_{1}\right|}\langle\eta\rangle^{2\left|\beta_{1}\right|}$. The integral in (5.3) satisfies thus

$$
\begin{aligned}
& \left|\int_{\eta} e^{i \eta \cdot x} \partial_{\xi}^{\beta_{1}}\left(e^{\tau\langle\xi+\eta\rangle^{\sigma}-\tau\langle\xi\rangle^{\sigma}}\right)(i \eta)^{\alpha} \partial_{\xi}^{\beta_{2}} \hat{a}(\eta, \xi) d \eta\right| \\
& \lesssim \int_{\eta}\left|\partial_{\xi}^{\beta_{1}}\left(e^{\tau\langle\xi+\eta\rangle^{\sigma}-\tau\langle\xi\rangle^{\sigma}}\right)\right||\eta|^{\alpha} e^{-\underline{\tau}\langle\eta\rangle^{\sigma}}\langle\xi\rangle^{m-\rho\left|\beta_{2}\right|}\left|F_{\xi, \beta_{2}}(\eta)\right| d \eta \\
& \lesssim\langle\xi\rangle^{m-\left|\beta_{1}\right|-\rho\left|\beta_{2}\right|} \int_{\eta} e^{\tau\langle\xi+\eta\rangle^{\sigma}-\tau\langle\xi\rangle^{\sigma}-\underline{\tau}\langle\eta\rangle^{\sigma}}\langle\eta\rangle^{2\left|\beta_{1}\right|+|\alpha|}\left|F_{\xi, \beta_{2}}(\eta)\right| d \eta .
\end{aligned}
$$

Next, we use inequality 2.9 in Lemma 2.2 to get

$$
\langle\eta\rangle^{2\left|\beta_{1}\right|+|\alpha|} \lesssim(\underline{\tau}-\tau)^{-\left(2\left|\beta_{1}\right|+|\alpha|\right) / \sigma} e^{(\underline{\tau}-\tau)\langle\eta\rangle^{\sigma}}
$$

hence

$$
\begin{aligned}
& \int_{\eta} e^{\tau\langle\xi+\eta\rangle^{\sigma}-\tau\langle\xi\rangle^{\sigma}-\underline{\tau}\langle\eta\rangle^{\sigma}}\langle\eta\rangle^{2\left|\beta_{1}\right|+|\alpha|}\left|F_{\xi, \beta_{2}}(\eta)\right| d \eta \\
& \lesssim(\underline{\tau}-\tau)^{-\left(2\left|\beta_{1}\right|+|\alpha|\right) / \sigma} \int_{\eta} e^{\tau\langle\xi+\eta\rangle^{\sigma}-\tau\langle\xi\rangle^{\sigma}-\tau\langle\eta\rangle^{\sigma}}\left|F_{\xi, \beta_{2}}(\eta)\right| d \eta
\end{aligned}
$$

and we conclude using the proof of Proposition 3.1 . 
We recall also the asymptotic expansion of $\widetilde{a}$, as given in Proposition 2.1 in [CNR].

Lemma 5.2 (Asymptotic expansion of $\widetilde{a}$ ). For any $k \in \mathbb{N}$ there holds

$$
\widetilde{a}(x, \xi)=\sum_{|\alpha| \leq k} \frac{i^{\alpha}}{\alpha !} \partial_{x}^{\alpha} a(x, \xi)\left(\tau \partial_{\xi}\langle\xi\rangle^{\sigma}\right)^{\alpha}+R
$$

with $R$ in $\mathrm{S}_{1,0}^{\max \{m-(k+1)(1-\sigma), m-2+\sigma\}}$.

This result is used in particular in our forthcoming papers [Mor17a] and Mor17b].

\section{References}

[BMM13] Jacob Bedrossian, Nader Masmoudi, and Clément Mouhot. Landau damping: paraproducts and Gevrey regularity. arXiv preprint arXiv:1311.2870, 2013.

[CNR] Ferruccio Colombini, Tatsuo Nishitani, and Jeffrey Rauch. Weakly hyperbolic systems by symmetrization. eprint arXiv:1508.03945v2.

[HR01] Chen Hua and Luigi Rodino. Paradifferential calculus in gevrey classes. Journal of Mathematics of Kyoto University, 41(1):1-31, 2001.

[Mor16a] Baptiste Morisse. On hyperbolicity and Gevrey well-posedness. Part 1: the elliptic case. arXiv preprint arXiv:1611.07225, 2016.

[Mor16b] Baptiste Morisse. On hyperbolicity and Gevrey well-posedness. Part 2: scalar or degenerate transitions. arXiv preprint arXiv:1611.08184, 2016.

[Mor17a] Baptiste Morisse. On hyperbolicity and Gevrey well-posedness. Part 3: a class of weakly hyperbolic systems. In preparation, 2017.

[Mor17b] Baptiste Morisse. On hyperbolicity and Gevrey well-posedness. Part 4: generic non-scalar transitions. In preparation, 2017.

[Rod93] Luigi Rodino. Linear partial differential operators in Gevrey spaces. World Scientific, 1993. 